\title{
USO DE DRONES EN LA SEGURIDAD VIAL DE LA CARRETERA 2402, SECTOR LA PLATA-LABERINTO, DEPARTAMENTO DEL HUILA*
}

\section{USE OF DRONES IN THE ROAD SAFETY OF THE ROAD 2402, SECTOR LA PLATA - LABERINTO, DEPARTMENT OF THE HUILA}

Recibido: 9 de agosto de 2018

Evaluado: 14 de noviembre de 2018

Aprobado: 9 de enero de 2019

\author{
Wilson Aldemar Santander-Derazo* \\ Universidad del Cauca \\ Orcid: 0000-0003-0922-8856
}

Cómo citar este artículo: Santander-Derazo, W. A. (2019). Uso de drones en la seguridad vial de la carretera 2402, sector La PlataLaberinto, departamento del Huila. Revista Estrategia Organizacional, 8 (1). doi: https://doi.org/10.22490/25392786.3175

\section{RESUMEN}

La problemática que se está presentando en temas de accidentalidad, se evidencian en las cifras de los accidentes de tránsito presentados en Colombia, catalogándolos como la primera causa de muerte en niños entre 5 y 14 años y la segunda causa de muerte entre adultos de 15 y 44 años. Esto ha hecho que el tema de la seguridad vial cobre gran importancia en Colombia. La investigación que se presenta en este artículo busca plantear una alternativa para determinar cuáles son los factores que afectan la seguridad vial mediante el uso de los drones. Por lo anterior, se ha tomado como referencia la carretera 2402, sector La Plata-Laberinto, localizada en el departamento del Huila, y se concibió la utilización de dichos aparatos aplicado a la seguridad vial.

Palabras clave: accidentalidad, factores viales, gestión, seguridad vial, carretera 2402 , drones.

\footnotetext{
* $\quad$ Artículo de investigación. Producto del trabajo realizado en el marco de la especialización en Gestión de Proyectos.

** Ingeniero Civil, Universidad del Cauca, Especialista en Gestión de Proyecto, UNAD.

Correo electrónico: wasd20@hotmail.com
} 


\begin{abstract}
The problem of accident rates is made evident by the figures related to traffic accidents in Colombia. They are categorised as the first cause of death in children between 5 and 14 years of age and the second cause of death among adults of 15 to 44 years. This has made the issue of road safety very important in Colombia. The research presented in this article seeks to propose an alternative to determine which factors, related to road safety, that could be improved through the use of drones. For the above, highway 2402, sector La Plata-Laberinto, located in the department of Huila, has been used as a reference and a plan for the use of such devices with the aim of improving road safety was conceived.
\end{abstract}

Keywords: Accident rates, Road factors, Road safety, Management, Highway 2402, Drones.

\section{ANTECEDENTES}

En Colombia las altas cifras de accidentalidad y la tendencia presentada en los últimos diez años (2005-2015), muestran 1.836.373 accidentes de tránsito en lo corrido del período, fenómeno que cobró la vida de 58.122 personas y dejó lesionadas a 411.957.

Tan solo en 2015 se presentaron 157.693 accidentes en los que fallecieron 6.352 personas, lo que supone un incremento del 2,1 \% frente al 2013, y 41.452 resultaron lesionadas, cifra que representa un aumento del 6,2\% con respecto a 2014.
Estas cifras evidencian que los accidentes de tránsito en Colombia son la primera causa de muerte en niños entre 5 y 14 años y la segunda causa de muerte en adultos entre 15 y 44 años. Además, los motociclistas ocupan el primer lugar en afectados y causantes de dichos accidentes, desplazando a los peatones de dicho indicador. (El Universal, 2015). Según los reportes de la Dirección de Tránsito y Transporte de la Policía Nacional, seccional Huila las cifras de accidentalidad para el año 2015, en la vía 2402; sector: La Plata-Laberinto, fueron las siguientes: 
Tabla 1. Reporte de accidentalidad año 2015 - vía 2402. Sector: La Plata - Laberinto.

\begin{tabular}{|l|l|l|l|}
\hline \multicolumn{5}{|c|}{ ACCIDENTALIDAD 2015} \\
\hline VÍA: 2402 & SECTOR: & \multicolumn{2}{l|}{ LA PLATA - LABERINTO } \\
\hline MES & ACCIDENTES & HERIDOS & MUERTOS \\
\hline ENERO & 2 & 10 & 0 \\
\hline FEBRERO & 1 & 3 & 1 \\
\hline MARZO & 3 & 0 & 0 \\
\hline ABRIL & 2 & 0 & 0 \\
\hline MAYO & 1 & 0 & 1 \\
\hline JUNIO & 1 & 0 & 0 \\
\hline JULIO & 2 & 2 & 0 \\
\hline AGOSTO & 1 & 1 & 0 \\
\hline SEPTIEMBRE & 0 & 0 & 0 \\
\hline OCTUBRE & 4 & 15 & 0 \\
\hline NOVIEMBRE & 2 & 2 & 0 \\
\hline DICIEMBRE & 3 & 2 & 2 \\
\hline \multicolumn{5}{|l|}{} \\
\hline TOTAL & $\mathbf{2 2}$ & $\mathbf{3 5}$ & $\mathbf{4}$ \\
\hline
\end{tabular}

Fuente: tomada de los registros de accidentalidad de la Administración vial 2 (HUI)

Las cifras anteriores son un claro reflejo de la necesidad de implementar sistemas de seguridad vial que permitan identificar los posibles sitios críticos, para evaluar las deficiencias, desde el punto de vista de la ingeniería civil enfocado a las carreteras, y entregar dichos documentos técnicos al INVIAS (Instituto Nacional de Vías) para que tome las medidas necesarias y así disminuir los índices de accidentalidad.

El uso de los drones no es nuevo, hace casi un siglo, el controvertido ingeniero inglés
Archibald Low (1888 - 1956) demostró ser capaz de controlar un pequeño y novedoso prototipo de biplano guiado por radio; sin embargo, años posteriores se utilizaron con fines militares (Consejería de economía y hacienda comunidad de Madrid, 2015). En el presente, los drones se pueden considerar como robots no antropomorfos con una inmensa autonomía de vuelo y una gran gama de posibilidades de aplicación. A la fecha no se encuentra información referente de trabajos enfocados a la seguridad vial mediante el uso de drones. 
CONTEXTO DONDE SE PRESENTA EL CONFLICTO

La Red Nacional de Carreteras es la red vial de Colombia regulada por el Ministerio de Transporte Colombiano mediante el Instituto Nacional de Vías (INVIAS) y sus direcciones territoriales y a veces delegadas a empresas privadas por concesión.

El sistema se compone por la Red Primaria, Red Secundaria y Red Terciaria. La Red de Carreteras colombiana es de $166.500 \mathrm{Km}$, de los que un $14 \%$ está pavimentado. Según el informe de la Cámara Colombiana de Infraestructura (2008), Colombia tiene $9 \mathrm{~km}$ de vías por cada kilómetro cuadrado de área (Tratado de libre comercio andino, EEUU).

La nomenclatura vial se adoptó mediante la Resolución 0000339 de 1999, en esta se estipula que las Rutas Nacionales se identifican con una señal en forma de escudo heráldico suizo - redondeado con tres puntas en el borde superior y una unta en el borde inferior- el cual tiene dentro otro escudo más pequeño y ancho de borde negro y en el centro se encuentra los dos dígitos que señalan el número de la ruta.

Las troncales (dirección sur-norte) se identifican con un número impar y las transversales (dirección occidente-oriente) con número par.
Las rutas tanto como los tramos, ramales, pasos y subramales pueden ser identificados mediante los postes de referencia al lado de la vía generalmente están instalados cada kilómetro.

Las rutas se dividen en tramos. Estos no son mayores de 150 kilómetros y su numeración se hará en forma continua del 01 en adelante, de sur a norte en las troncales y de occidente a oriente en las transversales. Los puntos de iniciación y terminación de cada tramo deben corresponder en lo posible a sitios o poblaciones de importancia. Los tramos se identifican con cuatro dígitos, los dos primeros corresponden al número de la ruta y los dos últimos al tramo considerado (INVIAS, 2016).

Por otra parte, las carreteras se definen de la siguiente manera:

- Carreteras primarias: Este tipo de carreteras pueden ser calzadas dividas según las exigencias propias de cada proyecto. Deben ser pavimentadas.

- Carreteras secundarias: Vías que unen las cabeceras municipales entre sí y/o que vienen desde una cabecera municipal y conectan con una carretera primaria. Pueden ser pavimentadas o en afirmado. 
- Carreteras terciarias: Vías que unen las cabeceras municipales con sus veredas entre sí. Deben ser en afirmado. Si se pavimentan deben cumplir a las condiciones geométricas fijadas para las vías secundarias.

La información anterior sirve como referencia para determinar las circunstancias que rodean el conflicto y una mejor comprensión del mismo, por lo tanto cuando se habla de la carretera 2402 , se hace referencia a la ruta transversal 24 y tramo 02, esta vía es secundaria debido a que se conecta con una carretera primaria la ruta 45; adicionalmente el sector a analizar es el comprendido entre el municipio de La Plata hasta Laberinto, en el departamento del Huila. Los postes de referencia correspondientes en este tramo inician en el PR 48 (La Plata) hasta el PR 100 (Laberinto), la longitud aproximada corresponde a 52,0 kilómetros de vía, los cuales están pavimentados con concreto asfáltico y un ancho promedio de calzada de 9,0 metros.

\section{CONFLICTO (NO CONFORMIDAD) QUE DA LUGAR AL DESARROLLO DEL PROYECTO}

Actualmente se puede hablar de cuatro factores principales que afectan la seguridad vial, entre los cuales encontramos:
1. Factores humanos, siendo esta una de las primeras causas de accidentalidad.

\section{Factores vehiculares.}

3. Factores de las vías.

4. Factores del medio.

El presente artículo busca determinar cuáles son los factores de la vía que afectan la seguridad vial, en este caso la vía 2402, la cual conduce desde el municipio de La Plata y conecta con la ruta 45 hacia Neiva, la capital del departamento del Huila. Esta es una vía de gran importancia, esencialmente por el gran flujo vehicular que se desplaza desde el departamento del Cauca y el occidente del Huila hacia el centro del departamento y del país. Cuenta con un tránsito promedio diario (TPD) de 998 vehículos por día de acuerdo con los conteos de 2010, con una composición de $64 \%$ automóviles, 4 \% de buses y $32 \%$ de camiones. En el año 2015, según los reportes de accidentalidad, se presentaron 22 accidentes de tránsito en este tramo, dejando como consecuencia 35 heridos y 4 muertos. Lo anterior refleja una situación adversa para los usuarios que transitan por esta vía, si bien es cierto que los accidentes pudieron haber sido provocados por alguno de los cuatro factores, nos centraremos en determinar aquellos que dependieron del factor vial (INVIAS, 2015). 


\section{DESCRIPCIÓN DEL PROBLEMA}

De acuerdo con lo plasmado anteriormente se puede decir que el problema consiste básicamente en los frecuentes accidentes de tránsito ocurridos en la vía 2402; sector La Plata-Laberinto, acompañado de la carencia de organismos públicos y privados encargados de prevenir y reducir los accidentes de tránsito que estén en función del factor vial. Es común encontrar entidades que se encargan de inspeccionar los sitios de accidentes y de llevar un control de estos, pero por la falta de conocimiento técnico no tienen la competencia suficiente para evaluar de forma más detalla la situación y brindar las posibles soluciones.

\section{COMITENTE, SPONSOR DEL}

\section{PROYECTO}

- Ingecol Santander S.A.S.

- Administración de Mantenimiento vial 03 - Huila.

\section{STAKEHOLDERS DEL PROYECTO}

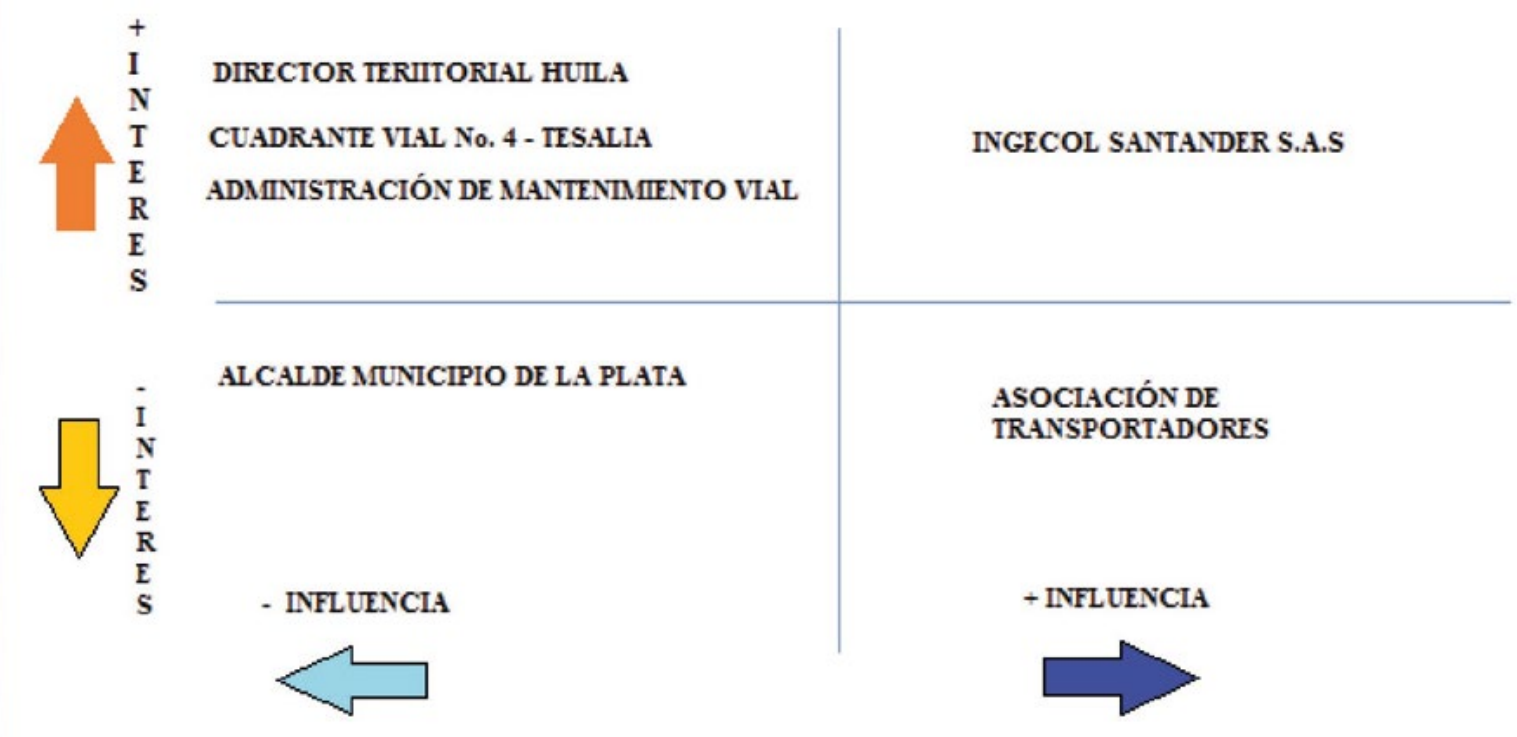

Figura 1. Stakeholders

Fuente: elaboración propia 


\section{POSIBLES MODALIDADES DE SOLUCIÓN DEL PROBLEMA}

Entregar un informe detallado al Instituto Nacional de Vías, acerca de las irregularidades encontradas en la vía a la cual se le realizará el estudio, para que tome medidas sobre el asunto, posteriormente hacer un seguimiento durante un período de hasta de seis meses para determinar si se tomaron las medidas necesarias por parte del INVIAS o el ente a cargo de la vía.

Entregar un estudio de señalización vertical al Instituto Nacional de Vías, en el cual se contemple el suministro y la instalación de señalización vertical informativa, preventiva y reglamentaria, en los sitios críticos identificados previamente, para que sea gestionado con recursos propios y así mitigar la accidentalidad.

Entregar un presupuesto de obra, en el cual se cuantifique el valor total del proyecto que se requiere para intervenir los sitios críticos que se identificaron inicialmente y que representan riesgos potenciales de accidentalidad, adjunto al presupuesto enviar un documento formal, solicitando dichos recursos para su pronta ejecución.

Realizar una reunión con los transportadores, policía de carreteras y funcionarios del INVIAS, socializar el proyecto aplicado, pactar compromisos entre las partes involucradas y mencionar las recomendaciones a las que haya lugar.

\section{CONSTRICCIONES Y RESTRICCIONES DEL PROYECTO}

Como constricciones seencuentra que los reportes de accidentalidad están incompletos. Igualmente, el tramo de la vía a estudiar es complejo.

Una de las principales restricciones es la de tipo financiero o presupuestal, actualmente el Instituto Nacional de Vías, entregó en concesión la vía principal que conduce desde Neiva a Mocoa, Ruta 45, lo que implica la reducción en los ingresos producto de los peajes, a su vez dicho impacto se ve reflejado en el resto de la red nacional de carreteas ya que no se cuenta con la misma disponibilidad presupuestal para ejecutar proyectos de mejoramiento y mantenimiento del resto de las vías.

Otra de las restricciones que se presentan es que la vía sobre la cual se pretende realizar dicho proyecto está a cargo del INVIAS, lo que implica que ninguna otra entidad ya sea pública o privada puede intervenir sobre ésta sin tener el permiso respectivo de uso de zona de vía, trámite que es muy dispendioso. 


\section{FORMULACIÓN Y SISTEMATIZACIÓN DEL PROYECTO}

\section{FORMULACIÓN DEL PROBLEMA}

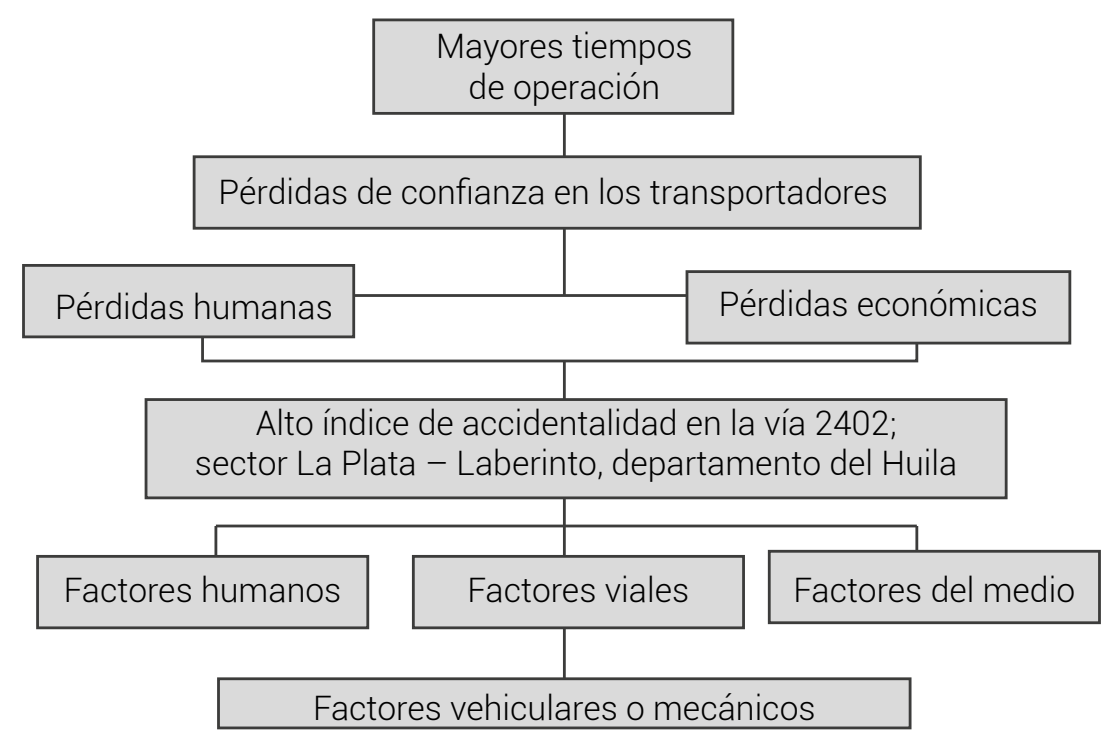

Figura 2. Árbol de problemas

Fuente: elaboración propia

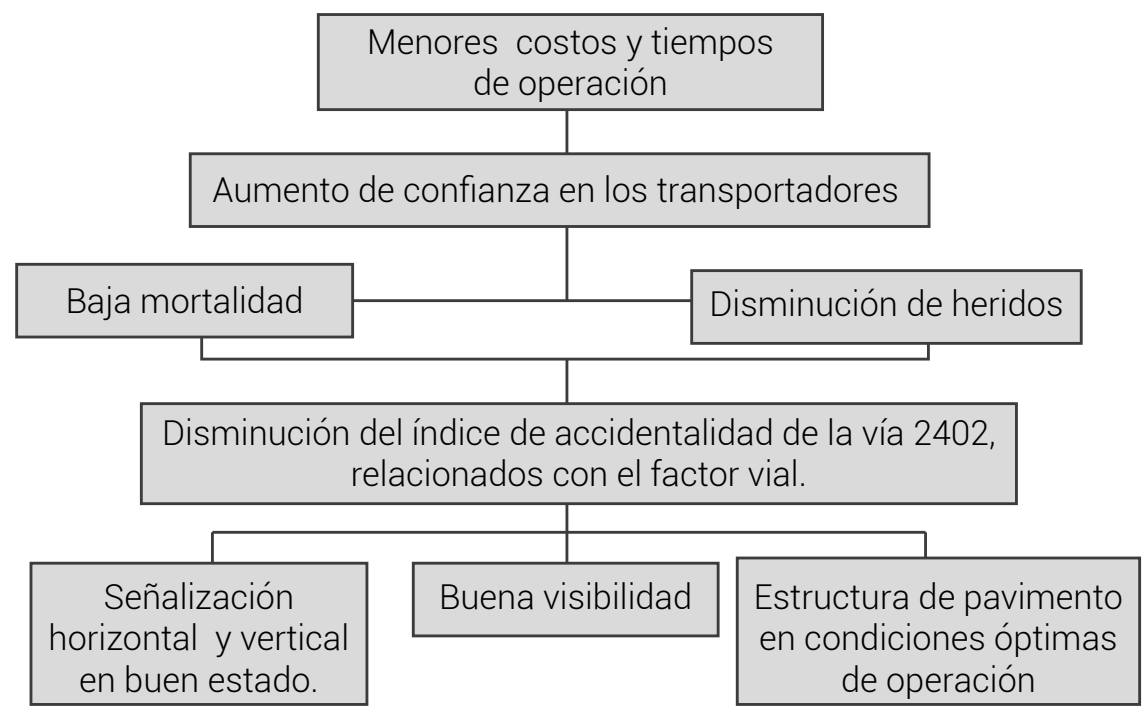

Figura 3. Árbol de objetivos

Fuente: elaboración propia 


\section{SISTEMATIZACIÓN DEL PROBLEMA}

Determinar qué propósito tiene la identificación de los factores viales relacionados con la accidentalidad de la vía 2402 , sector La Plata - Laberinto, con el fin de establecer soluciones técnicas y prácticas de tal manera que se logre crear un antecedente en el campo de la seguridad vial mediante el uso de drones, generando así un impacto ante entidades públicas y privadas en caso de fijar la viabilidad del proyecto.

Caso de negocio. El presente proyecto aplicado es viable en términos de la inversión requerida, porque no solo está en juego el factor económico sino también la integridad física de los usuarios que transitan por las vías.

En término de costos la inversión que se requiere es relativamente baja si se compara con los resultados esperados, adicionalmente reviste de gran importancia desarrollar este proyecto en un tramo comprendido en aproximadamente 52 kilómetros, el cual cuenta con un Tránsito Promedio Diario de 998 vehículos por día según los conteos de 2010, y así ser utilizado como referencia a nivel nacional para su aplicación en tramos mucho más largos, ya que actualmente no se tiene conocimiento de trabajos similares.
Factores ambientales de la empresa. Los factores que influyen en el desarrollo del acta de constitución del proyecto son los siguientes:

- Resolución 1384 de 2010. Límites de velocidad en las carreteras nacionales, departamentales, distritales y municipales de Colombia.

- Manual de señalización vial 2015. Dispositivos para la regulación de tránsito en calles, carreteras y ciclorutas de Colombia.

- Manual para la inspección visual de pavimentos flexibles 2006.

- Especificaciones generales de construcción de carreteras 2013 - INVIAS 2013.

- Circular reglamentaria para los R/PAS o drones No. 002 - Aeronáutica civil y la Fuerza Aérea.

- Condiciones de uso de drones:

- Licencia de piloto privado con curso en tierra que dura 6 meses.

- 40 horas de vuelo y 200 despegues y aterrizajes, previos, certificados por la escuela de aviación.

- Identificación y matrícula del dron. 
- Póliza de seguro para daños a terceros.

- Solicitud con plan de vuelo ante la Aeronáutica Civil con 15 días hábiles de anticipación.

○ El dron debe tener un color que permita ser identificado fácilmente en el aire.

\section{EVALUACIÓN DE FACTIBILIDAD ECONÓMICA DEL PROYECTO}

Para determinar la factibilidad económica del proyecto se presenta el siguiente análisis costo beneficio:

Tabla 2. Análisis Beneficio / Costo

\begin{tabular}{|c|c|c|c|c|c|}
\hline \multicolumn{6}{|c|}{ ANÁLISIS DE COSTO - BENEFICIO : COSTOS } \\
\hline $\begin{array}{l}\text { PROGRAMA DE COSTOS ETAPA } \\
\text { EJECUCIÓN DEL PROYECTO }\end{array}$ & mes 0 & mes 1 & mes 2 & mes 3 & mes 4 \\
\hline Recolección de información & \$ - & $\$ 6.720 .000$ & $\$$ & $\$$ & $\$$ \\
\hline Análisis de información & $\$-$ & $\$ 1.680 .000$ & $\$ 1.680 .000$ & $\$$ & $\$$ \\
\hline Trabajo de campo & $\$-$ & $\$-$ & $\$ 8.568 .000$ & $\$ 3.672 .000$ & \\
\hline Trabajo de oficina & \$ - & S - & $\$$ & $\$ 4.752 .000$ & $\$ 3.168 .000$ \\
\hline Cierre del proyecto & $\$-$ & $\$-$ & $\$$ & $\$$ & $\$ 3.360 .000$ \\
\hline Costo total por mes & \$- & $\$ 8.400 .000$ & $\$ 10.248 .000$ & $\$ 8.424 .000$ & $\$ 6.528 .000$ \\
\hline $\begin{array}{l}\text { Costo Total Ejecución del } \\
\text { Proyecto }\end{array}$ & \multicolumn{2}{|c|}{$\$ \quad 33.600 .000$} & & & \\
\hline
\end{tabular}

\begin{tabular}{|c|c|c|c|c|c|}
\hline \multicolumn{6}{|c|}{ ANÁLISIS DE COSTO - BENEFICIO : COSTOS } \\
\hline \multirow{2}{*}{$\begin{array}{l}\text { PROGRAMA DE COSTOS } \\
\text { PREVISTOS }\end{array}$} & \multicolumn{5}{|c|}{ Año fiscal } \\
\hline & AÑO 0 & AÑO 1 & AÑO 2 & AÑO 3 & AÑO 4 \\
\hline Costos de Ejecución de proyecto & $\$-$ & $\begin{array}{l}\$ \$ \\
33.600 .000,00\end{array}$ & $\$$ & $\$$ & $\$$ \\
\hline Costos de Implementación & $\$-$ & & $\begin{array}{l}\$ \\
540.000 .000,00\end{array}$ & $\begin{array}{l}\$ \\
420.000 .000,00\end{array}$ & $\begin{array}{l}\$ \\
360.000 .000,00\end{array}$ \\
\hline Costos de mantenimiento & $\$-$ & $\$-$ & $\$$ & & $\begin{array}{l}\$ \\
36.000 .000,00\end{array}$ \\
\hline Costo Total por año & \$ - & $\$ 33.600 .000$ & $\begin{array}{l}\$ \\
540.000 .000\end{array}$ & $\begin{array}{l}\$ \\
420.000 .000\end{array}$ & $\$ 396.000 .000$ \\
\hline Costo Total del Programa & $\$ 1.38$ & 9.600 .000 & & & \\
\hline
\end{tabular}




\begin{tabular}{|c|c|c|c|c|c|}
\hline \multicolumn{6}{|c|}{ ANÁLISIS DE COSTO - BENEFICIO : BENEFICIOS } \\
\hline \multirow{2}{*}{ FUENTES DE BENEFICIO } & \multicolumn{5}{|c|}{ Año fiscal } \\
\hline & AÑO 0 & AÑO 1 & AÑO 2 & AÑO 3 & AÑ̃ 4 \\
\hline Reducción de costos de Transporte & $\$-$ & $\$$ & $\begin{array}{l}\$ \\
50.000 .000,00\end{array}$ & $\begin{array}{l}\$ \\
50.000 .000,00\end{array}$ & $\begin{array}{l}\$ \\
50.000 .000,00\end{array}$ \\
\hline Ingresos mejorados & $\$-$ & $\$-$ & $\begin{array}{l}\$ \\
200.000 .000,00\end{array}$ & $\begin{array}{l}\$ \\
200.000 .000,00\end{array}$ & $\begin{array}{l}\$ \\
200.000 .000,00\end{array}$ \\
\hline mejor calidad de vida - empleo & $\$-$ & $\$-$ & $\begin{array}{l}\$ \\
378.000 .000,00\end{array}$ & $\begin{array}{l}\$ \\
294.000 .000,00\end{array}$ & $\begin{array}{l}\$ \\
252.000 .000,00\end{array}$ \\
\hline disminución de vehículos averiados & $\$-$ & $\$-$ & $\begin{array}{l}\$ \\
250.000 .000,00\end{array}$ & $\begin{array}{l}\$ \\
250.000 .000,00\end{array}$ & $\begin{array}{l}\$ \\
250.000 .000,00\end{array}$ \\
\hline Beneficio Total por año & \$ - & & \begin{tabular}{|l|}
$\$$ \\
878.000 .000 \\
\end{tabular} & \begin{tabular}{|l|}
$\$$ \\
794.000 .000 \\
\end{tabular} & $\$ 752.000 .000$ \\
\hline Total de Beneficios & $\$ 2.4$ & 4.000 .000 & & & \\
\hline
\end{tabular}

\begin{tabular}{|c|c|c|c|c|c|}
\hline \multicolumn{6}{|c|}{ ANÁLISIS DE COSTO - BENEFICIO : RESUMEN } \\
\hline \multirow{2}{*}{ FLUJO DE CAJA LIBRE } & \multicolumn{5}{|c|}{ Año fiscal } \\
\hline & AÑO 0 & AÑO 1 & AÑO 2 & AÑO 3 & AÑO 4 \\
\hline Costos & \$ - & $-\$ 33.600 .000$ & $\begin{array}{l}-\$ \\
540.000 .000\end{array}$ & $-\$ 420.000 .000$ & $-\$ 396.000 .000$ \\
\hline Beneficios & $\$-$ & $\$-$ & $\$ 878.000 .000$ & $\$ 794.000 .000$ & $\$ 752.000 .000$ \\
\hline Flujo Neto de efectivo & \$ - & $-\$ 33.600 .000$ & $\begin{array}{l}\$ \\
338.000 .000\end{array}$ & $\begin{array}{l}\$ \\
374.000 .000\end{array}$ & $\$ 356.000 .000$ \\
\hline Factor de descuento & 1,0000 & 0,9302 & 0,8653 & 0,8050 & 0,7488 \\
\hline \multicolumn{6}{|l|}{ Flujos de descuento } \\
\hline Costos & $\mathbf{\$}-$ & $-\$ 31.255 .814$ & $-\$ 467.279 .611$ & $-\$ 338.083 .439$ & $-\$ 296.525 .010$ \\
\hline Beneficios & $\$-$ & $\$-$ & $\$ 759.762 .034$ & $\$ 639.138 .692$ & $\$ 563.097 .998$ \\
\hline Valor neto descontado & \$ - & $-\$ 31.255 .814$ & $\begin{array}{l}\$ \\
292.482 .423\end{array}$ & \$ 301.055 .253 & \$ 266.572.989 \\
\hline Valor neto acumulado & & $-\$ 31.255 .814$ & $\$ 261.226 .609$ & $\$ 562.281 .862$ & $\$ 828.854 .851$ \\
\hline
\end{tabular}




\begin{tabular}{|l|l|l|}
\hline TASA DE DESCUENTO & $\mathbf{7 , 5 \%}$ & \\
\hline & & \\
\hline VAN & $\mathbf{\$ 8 2 8 . 8 5 4 . 8 5 1}$ & !Es rentable $\boldsymbol{i}$ \\
\hline & & \\
\hline TIR & $10,14 \%$ & \\
\hline & & \\
\hline B / C & $\mathbf{1 , 7 4}$ & !Es rentable \\
\hline
\end{tabular}

La anterior evaluación económica muestra que el proyecto es rentable, aunque el impacto económico durante los cuatro años no es muy significativo, existe un factor indispensable y es el factor humano el cual es fundamental a la hora de tomar decisiones definitivas sobre la implementación del proyecto. Por otra parte la tasa interna de retorno está por encima de a la tasa de descuento aplicada al proyecto lo que representa que el proyecto brinda un buen margen de seguridad en el aspecto económico siempre y cuando la tasa de descuento no suba y en caso de ser así, para que el proyecto siga siendo favorable tendría que llegar hasta el punto de equilibrio, de lo contrario el proyecto podría verse destinado a ser desfavorable.

Los costos asumidos en la fase de implementación y mantenimiento están basados en mi experiencia en el campo de la ingeniería civil aplicada a proyectos de mejoramiento y mantenimiento de vías y de administración vial en esta zona.

\section{EVALUACIÓN SOCIAL}

La parte inicial del proyecto, que consiste en la elaboración de estudios y diseños a partir de la identificación de sitios críticos relacionados con accidentes de tránsito en la vía en estudio, generará un bajo impacto social debido al poco personal e involucrados durante el desarrollo del mismo, pero existe una gran posibilidad de generar un impacto mucho mayor cuando dichos estudios se efectúen y se tengan resultados concretos debido a las demandas que los usuarios podrían interponer ante la entidad pública a la cual le corresponda este tramo de vía, en este caso en específico al INVIAS territorial Huila.

La implementación de las obras en pro de mejorar las condiciones de seguridad vial también tendría un gran impacto positivo para los usuarios de la vía, debido a la generación de empleo para la ejecución de las obras, aumentaría el flujo vehicular con ganancias significativas para el sector transporte, y de hecho los costos por reparaciones o accidentes serían mucho menores. 


\section{DIAGRAMAS DE RED DEL CRONOGRAMA DEL PROYECTO}

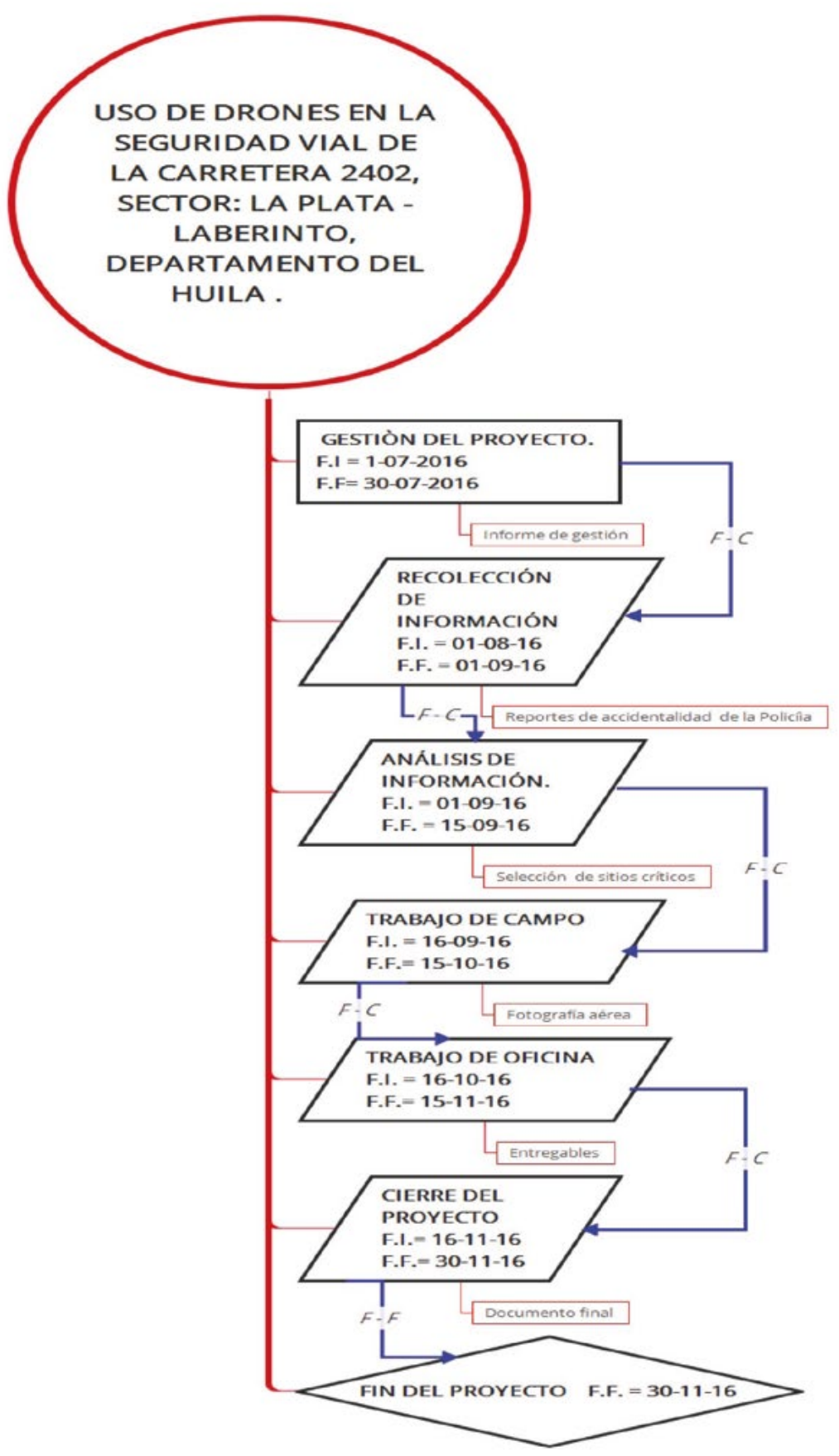

Figura 3. Diagrama de red del cronograma del proyecto

Fuente: elaboración propia 


\section{CONCLUSIONES}

La importancia de un plan de gestión de riesgos en un proyecto siempre será vital porque los riesgos nunca terminarán, los que surgen dejan de existir o se mitigan y aparecerán nuevos riesgos, los proyectos siempre traen nuevos conocimientos y nuevas experiencias sobre sus riesgos y formas de tratarlos o gestionarlos que de una forma correcta estos riesgos pueden convertirse en oportunidades de mejora o avance para la organización. La identificación de los mismos es un medio por el cual se determina qué riesgos pueden afectar al proyecto y es indispensable documentar sus características. Este es un proceso interactivo, ya que se descubrirán nuevos riesgos a medida que se avance con el ciclo de vida del proyecto por tal motivo es de gran importancia que los profesionales encargados de ejecutar el proyecto estén atentos a los riesgos futuros que se pueden presentar en el proyecto durante la etapa de ejecución y brindar las posibles soluciones.

En el presente proyecto la cuantificación de los riesgos se desarrolló durante el análisis de riesgos, siendo este método uno de los más utilizado en la toma de decisiones en proyectos empresariales, los gerentes o directores de empresa se apoyan en su juicio, experiencia e intuición para la toma de decisiones; dicho análisis y gestión de riesgos permitirá que el proyecto se ejecute dentro de los tiempos establecidos y los costos previstos.

La estimación de costos no es una actividad estática, sino que cambia en diferentes puntos del ciclo de vida del proyecto por lo tanto no es posible predecir el comportamiento absoluto del mismo, de ahí la importancia de manejar y aplicar los conceptos tales como curva " $S$ ", variación del programa y de costos, $E V, A C, B A C, E T C$, entre otros, los cuales deben ser tenidos en cuenta para un mejor control del proyecto con el fin de proponer la solución más adecuada y poder llevar a cabo con éxito este proyecto.

Con el desarrollo del presente proyecto las entidades públicas y privadas, además de encontrar una alternativa de solución al problema de accidentalidad de la vía principal de salida hacia la capital del Huila; establece un marco referencial para la preparación de proyectos de inversión pública utilizando la metodología PMI. El mismo determina el proceso de Iniciación y Planificación de un Proyecto de consultoría, brindando las herramientas básicas para su seguimiento y control.

\section{REFERENCIAS}

Agüero Seas, W. (2007). Modelo para la Evaluación (Financiera, económica y ambiental) de proyectos. 
https://doi.org/10.22490/issn.2539-2786

Recuperado en: http://biblioteca.icap.ac.cr/BLIVI/ TESIS/2007/te_aguero_willian_2007.pdf

Alsina, J. (2009). Gestión del Valor Ganado "EVM" para control de proyectos v.2. Recuperado en: http:// www.liderproyecto.com/evmV3_necesidad_sistema_de control/que_es_la_tecnica_EVM.html

Arias, C., Ayala, J., Novoa, A. y Vega, F. (2016). Gestión de stakeholders: Trabajo colaborativo momento III. Colombia: Universidad Nacional Abierta y a Distancia.

Análisis de Riesgos. (s.f.). La Suma de Todos. Comunidad de Madrid. Recuperado en: http://www.madrid.org/ cs/StaticFiles/Emprendedores/Analisis_Riesgos/ pages/pdf/metodologia/4AnalisisycuantificaciondelRiesgo(AR)_es.pdf

Burbano, J. (2005). Presupuestos enfoque de gestión, planeación y control de recursos. Colombia: Ed Mc Graw Hill.

Figueroa Delgado, L. O. (2009). Módulo: Administración financiera. (s.d.).

Hernández, A. (s.f.). Formulación y evaluación de proyectos de inversión, Ed. Thomson.

INVIAS (Instituto Nacional de Vías). (s.f.). Documentos Técnicos. Cartilla de conteos vehiculares.
Recuperado en: http:// www.invias.gov.co/documentostecnicos/ cartilladeconteosvehiculares. pdf

INVIAS (Instituto Nacional de Vías). (s.f.). Documentos Técnicos. Resolución No. 0000339 de 199. Recuperado en: http:// www.invias.gov.co/documentostecnicos/resoluciones.pdf

Lopez Dumrauf, G. (2003). Técnicas de Evaluación de Proyectos de Inversión, Calculo Financiero Aplicado, Un enfoque Profesional. Recuperado en: http://datateca.unad.edu.co/contenidos/108002/Curso_2015/ Entorno_de_Conocimiento/Unidad_4/Evaluacion_ de_proyectos_de_inversion.pdf

Miranda Miranda, J. J. (s.f.). Gestión de Proyectos. Evaluación Financiera. Recuperado en: http://antioquia.gov.co/antioquia-v1/organismos/planeacion/ descargas/banco_proyectos/libro/9_evaluacion_financiera.pdf

Monreal, C. (2015). Plantillas para el gestor de proyectos. Gestión por Proyectos. Recuperado en: https:// gestionporproyectos.wordpress.com/plantillas-para-el-gestor-de-proyectos/

Normas APA. (s.f.). Sexta Edición. Recuperado en: http:// portales.puj.edu.co/ftpcentroescritura/ Recursos \%20C.E/Estudiantes/Referencia\%20bibliogr\%C3\%A1ficas/ Normas\%20APA\%20Sexta\% 20Edici\%C3 \%B 3n.pdf 
Project Management Institute. (2013). A guide to the Project Management Body of Knowledge (PMBOK Guide). (w.i.).

Rodriguez, R. (2015). Gestión de recursos humanos.

Slideshare. Recuperado en: http://es.slideshare.net/ RubenPrado1/07-gestion-de-recursos-humanos
Universidad Nacional Abierta y a Distancia-UNAD. (2011). Escuela de Ciencias Administrativas Contables Económicas y de Negocios Contenido didáctico del curso Matemáticas Financieras. Colombia.

Zelada, R. (2013). Gestión de Interesados - Guía del PMBOK. Slideshare. Recuperado en http://www.slideshare. net/RocioZeladaPMP/gestion-de-interesados -guia-del-pmbok-5ta-version?next_slideshow=1 